\title{
SUR UN ACROTHECIUM ISOLE DU « MAL DEL PINTO » MEXICAIN, ACROTHECIUM NIGRUM n. sp.
}

\author{
Par R. CIFERRI
}

Origine de la souche. - Nous avons recu la culture de ce champignon de M. le Prof. Ochoterena (voir note 1, p. 511), qui isola cette souche de la peau d'un homme atteint de " pinta negra " (pinta noire). La photographie reproduite dans la fig. 1 représente l'affection dans la main et le bras. Nous n'avons point reçu d'autres indications, mais M. Ochoterena a isolé aussi un deuxième champignon levuriforme, associé au précédent et qui 'sera publiế prochainement ( $\mathrm{n}^{\circ}$ 176).

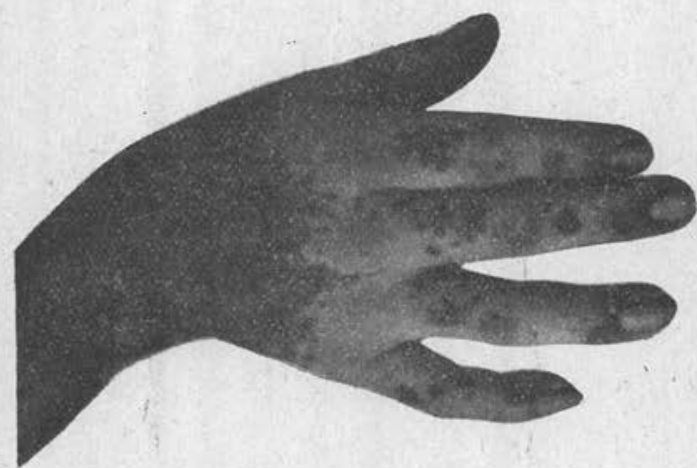

FIG. 1. - Plaques de la « pinta » noire mexicaine de la main et de l'avant-bras. Cliché de I. Ochoterena.

Aspect du champignon dans la lésion. - Nous ne connaissons rien sur ce sujet.

Caractères culturaux. - Nous limitons cette étude aux cultures sur la gélose pepto-glycosée de Sabouraud, car les autres n'offrent pas assez de particularités distinctives, en dehors d'un développement plus ou moins abondant.

Sur gélose pepto-glycosée de Sabouraud (formule originale; $p \mathrm{H}=6,8)$, le développement est rapide et envahissant ; il se forme un duvet noir, fuligineux, qui s'accroît en quelques jours jusqu'à

Annales de Parasitologie, $\mathrm{T}$. VII, $\mathrm{N}^{\circ} 6 .-1^{\mathrm{er}}$ novembre 1929, p. 524-535. 
couvrir complètement la surface libre. Cà et là, particulièrement après le premier développement, on voit des nuances gris-noires, peu distinctes. La colonie croît ensuite en s'épaississant, jusqu'à former un duvet pouvant atteindre $10 \mathrm{~mm}$. d'épaisseur. C'est alors qu'on peut voir des petites touffes nuancées de blanc, formées de mycélium stérile ou très peu fructifié. Le milieu de culture est, dès les premiers moments, et parallèlement à la croissance du champignon, pigmenté en noir ; le pigment se répand dans le substratum, jusqu'au revers de la culture, si elle n'est pas trop épaisse. Cette particularité, plus ou moins visible dans presque tous les milieux essayés, est plus accentuée dans les milieux riches en hydrates de carbone, le glycose et le maltose étant spécialement favorables.

Nous avons essayé plusieurs autres milieux, naturels et synthétiques : le développement du champignon est abondant sur gélose au bouillon de carotte, gélose aux prunes, gélose au moût de bière, etc., moins abondant sur pomme de terre ou gélose à la farine de maïs, etc. Dans le Raulin gélosé, et, en général, dans les milieux synthétiques, il y a un développement moins abondant, et aussi une production plus difficile (ou diffusion ?) du pigment noir. La liquéfaction de la gélatine est lente et tardive. Dans les milieux liquides, le développement est presque toujours plus lent et moins abondant "; quelquefois il y a diffusion d'un pigment jaune-brunâtre.

Caractères morphologiques. - Cette étude est faite d'après les cultures sur gélose pepto-glycosée de Sabouraud. Nous avons étudié ce champignon de la mème façon que Grubyella ochoterenai (1).

Mycélium. - Le mycélium est hyalin au début, mais presque aussitôt la couleur vire au foncé puis au noir. Cependant on peut voir des hyphes toujours blanches.

L'allongement des hyphes est rapide, et leur ramification abondante et irrégulière. Au commencement, elles sont de petit calibre, mais la membrane s'épaissit rapidement et parallèlement au développement de la couleur. On voit fréquemment des cloisons, surtout sur les hyphes pas trop jeunes. Une forme très fréquente d'hyphes septées est reproduite et schématisée dans la figure 2, B. Quelquefois la ramification est multiple en partant d'une même hyphe, qui forme un article terminal plus foncé et de forme irrégulière (C, fig. 2). Les hyphes simples mycéliennes sont rares, proportionnellement à la quantité d'hyphes toruleuses foncées ou

(1) Voir ces Annales, VII, 1929, p. 511-523. 
noires ; on voit tous les types d'hyphes monilioïdes. Les dimensions des éléments sont, naturellement, très variables. Nous croyons inutile de décrire en détail les différents types, car ils sont à peu près les mêmes chez presque toutes les Dematiées cultivées, d'une certaine complication morphologique. La fig. 2, A donne une idée d'un nœud mycélien isolé et vu au microscope.

Conidiophores. - Les conidiophores sont presque-toujours les rameaux latéraux des hyphes assez jeunes, dressés ou presque dressés (rarement rampants), quoique l'on puisse voir aussi se former des capitules conidiens aux extrémités des jeunes hyphes. Leur couleur est presque hyaline, mais peut devenir plus ou moins foncée. Ils peuvent être ramifiés ou non, avec tous les rameaux fertiles ou quelques-uns seulement; mais les ramifications sont toujours peu abondantes et limitées, en général, à quelques petits conidiophores secondaires latéraux. Aussi, dans ce cas, les capitules sont peu riches en conidies, presque toujours avec une ou deux conidies seulement. La longueur des conidiophores est très variable, même lorsqu'ils ne portent qu'une ou deux conidies. Le diamètre est de même très variable.

Conidies. - Les conidies sont normalement en capitules, et typiquement acrogènes, quoique l'on puisse voir exceptionnellement des conidies isolées ou en verticilles très réduits mais pleurogènes. L'insertion des conidies sur les rameaux fertiles est formée par une série de denticules très rapprochés au sommet du conidiophore (D, fig. 2) : cette dentelure, toujours peu évidente, peut aussi manquer. S'il y a un seul capitule conidien, généralement il est polyspore et porte de 2 à 12 conidies ou quelquefois plus encore ; plus fréquemment les capitules sont composés de 4 à 6 conidies, mais leur nombre est très variable, selon l'âge de la culture et surtout selon la composition et la richesse du milieu. Dans la fig. 2, F nous avons reproduit, en les schématisant, des capitules et des conidies isolées avec leur conidiophore et quelques hyphes mycéliennes. La couleur des conidies, presque hyaline lorsqu'elles sont très jeunes, devient ensuite très foncée. Cependant, une caractéristique presque constante c'est la couleur plus foncée, même presque noire, des portions centrales des conidies, soit d'une ou de deux cellules, selon que les conidies sont bi ou tri-septées. $\mathrm{Si}$ la conidie est seulement uni-septée, presque toujours les deux cellules sont à peu près de la même couleur. Cette différence de coloration est saisissante, et rappelle celle des conidies de Pestalozzia.

Le nombre des cloisons varie de 0 à 3 , car elles se forment progressivement; les conidies très jeunes sont continues. 
Sur 200 conidies, nous avons relevé les chiffres suivants :

\begin{tabular}{||c|c|c|c|c|}
\hline \hline Nombre de cloisons......... & 0 & 1 & 2 & 3 \\
\hline Fréquence................... & 10 & 24 & 39 & 127 \\
\hline
\end{tabular}

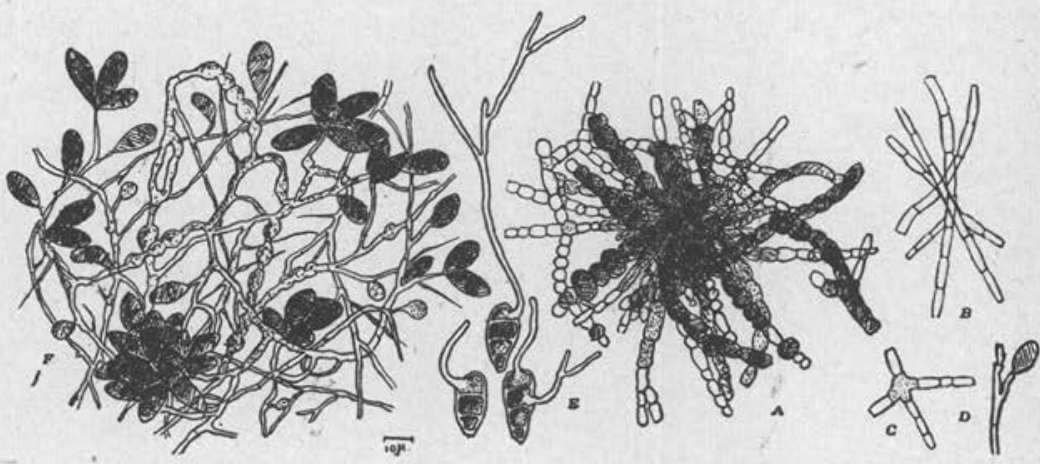

Fıg. 2. - Acrothecium nigrum n. sp. $a$, les deux types de mycélium ; $b$, mycélium simple, hyalin; $c$, nœud mycélien de passage entre deux types; $d$, extrémité denticulée d'un conidiophore, avec l'insertion d'une conidie; $e$, germination des conidies; $f$, aspect d'une touffe vue au microscope : mycélium, conidiophores ct capitules conidiens.

La forme des conidies est très variable aussi. Elles ont généralement la forme d'une ellipse plus ou moins allongée et peuvent être quelquefois ovoïdes ou piriformes. Il y a aussi des conidies un peu courbées, mais jamais falciformes. Très fréquentes sont aussi les formes aberrantes ; dans la figure $6, \mathrm{~A}$ on en voit quelques-unes, et dans la figure $6, \mathrm{~B}$ on voit les formes normales les plus fréquentes. L'extrémité libre de la conidie est presque toujours arrondie, ou un peu apiculée dans les conidies piriformes; l'extrémité d'insertion est presque toujours plus ou moins rectiligne. Leurs dimensions varient entre 6-11 $\mu$ de longueur sur $16-30 \mu$ de diamètre ; les dimensions les plus fréquentes sont $8-9$ sur $22-26 \mu$, et la moyenne 8 sur $24 \mu$; voici les résultats de la mensuration de 200 conidies : 


\begin{tabular}{|l|l|l|l|l|l|l|l|l|l|l|l|l|l|l|l|l||}
\hline Longueur en $\ldots .$. & 16 & 17 & 18 & 19 & 20 & 21 & 22 & 23 & 24 & 25 & 26 & 27 & 28 & 29 & 30 \\
\hline Fréquence...... & 1 & 1 & 3 & 4 & 4 & 3 & 25 & 28 & 69 & 16 & 19 & 11 & 8 & 5 & 3 \\
\hline \hline Diamètre en $\mu . .$. & 6 & 7 & 8 & 9 & 10 & 11 & & & & & & & \\
\hline Fréquence...... & 3 & 23 & 91 & 64 & 15 & 4 & & & & & & & & \\
\hline
\end{tabular}

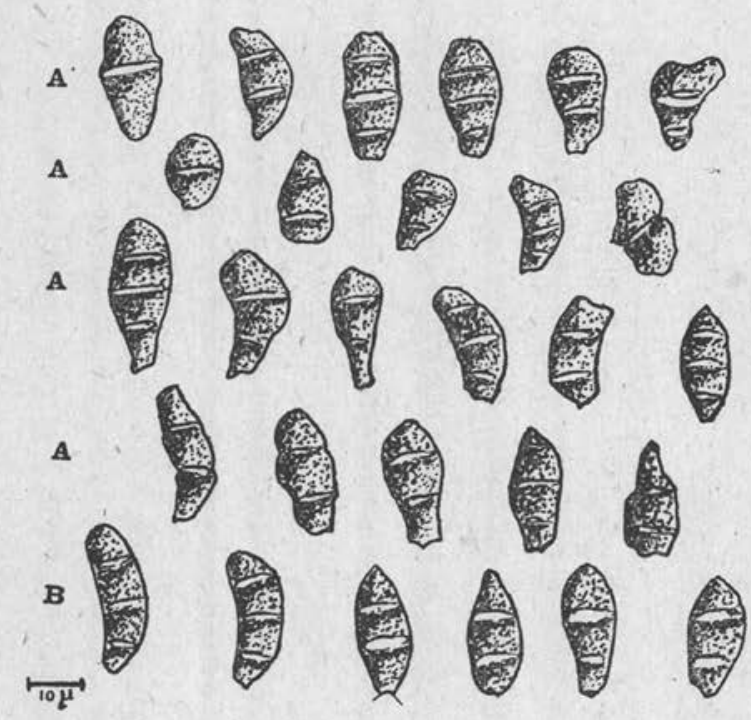

Fig. 3. - Conidies de l'Acrothecium nigrum n. sp. $\boldsymbol{a}$, formes aberrantes ; $b$, formes normales.

Développement du champignon. - La microculture en milieu liquide est un procédé peu favorable, car la croissance du mycélium est difficile et il ne se forme de rares conidies qu'à la surface exposée à l'air.

La microculture sur milieu solide (gélose pepto-glycosée) donne de meilleurs résultats. Le développement du mycélium et l'apparition des formes torulées est rapide; les éléments épaissis, isolés après leur formation, peuvent fonctionner comme chlamydospores, et germer sur place, en donnant d'autres hyphes et des conidiophores. Ces derniers ne sont pas clairement différenciés 
jusqu’à la formation des capitules conidiens. Les conidies apparaissent progressivement. Elles sont d'abord presque hyalines et continues, puis deviennent plus foncées et se cloisonnent. La différenciation de la couleur des cellules centrales est le dernier phénomène qu'on observe ; cependant des conidies de couleur uniforme peuvent germer également lorsqu'elles sont placées dans des conditions favorables. Nous avons déjà noté que la formation des capitules à nombreuses conidies est limitée aux conidiophores non ramifiés; les rameaux latéraux éventuels, presque toujours courts et minces, ne portent qu'une ou deux conidies.

Nous n'avons jamais vu germer sur place des conidies; lorsqu'elles sont isolées, elles germent très aisément sur bouillon glycosé ou sur gélose glycosée avec formation d'un ou plusieurs (deux ou trois) tubes germinatifs qui s'allongent et se ramifient rapidement. La cellule qui germe peut être, indifféremment, une des cellules centrales ou une de celles des extrémités. Trois conidies en germination sont représentées ( $\mathrm{E}$, fig. 2).

Position systématique du champignon. - Etant donnée la couleur sombre de ce champignon et ses capitules de conidies cloisonnées, il doit prendre place, parmi les Hyphomycètes, dans le groupe des Dematiées-Phæophragmiées-Acrotheciées et dans le genre Acrothecium. Nous allons esquisser brièvement l'histoire de ce genre, en partie à l'aide de l'excellent travail de M. Mason consacré à l'étude de l'Acrothecium lunatum Wakker.

Corda (1838) a créé le nom d'Acrothecium, comme sous-genre de Trichothecium, pour le Trichothecium parasitans Corda (1), placé d'abord parmi les Mucedinées.

Treize ans plus tard, Preuss (1851) employa le terme Acrothecium comme nom générique pour l'A. multisporum, qui possède des conidiophores sombres, des conidies elliptiques allongées, sub-hyalines et 1-3 septées. En même temps, Preuss créa aussi le genre Cacumisporium pour le $C$. tenebrosum, avec conidies obscures, 3 septées, plus ou moins acrogènes et verticillées.

Saccardo (1882) rapporte le Trichothecium (Acrothecium) parasitans Corda au genre Blastotrichum Corda, et considère Cacumisporium comme synonyme d'Acrothecium; Cacumisporium tenebrosum Preuss devient done Acrothecium tenebrosum (Preuss) Sacc., et se place au voisinage de A. bulbosum Sacc. Saccardo (1886) a considéré l'Acrothecium multisporum Preuss comme

(1) Ne pas confondre avec le genre Acrotheca Fuckel, également Dematiêe, mais de la section des Amerosporés (non Phragmosporés).

Annales de Parasitologie, T. VII, $\mathrm{N}^{\circ} 6 .-1^{\mathrm{er}}$ novembre 1929. 34. 
appartenant au genre Acrotheca Fuckel et il a donné comme type du genre Acrothecium le champignon $\mathrm{N}^{\circ} 99$ des "Champignons d'Hoyerswerda » de Preuss au lieu du $\mathrm{N}^{\circ} 36$, le $\mathrm{N}^{\circ} 99$ étant une espèce du genre Cordana Preuss. Saccardo transféra d'abord les trois espèces du genre Cordana dans le genre Acrothecium, mais plus tard, dans le Sylloge, il conserva dans Cordana une espèce (C. pauciseptata Preuss) et dans Acrothecium les deux autres (C. polyseptata Preuss et C. pedunculata Preuss).

La conclusion donnée par Mason est que le nom générique Acrothecium Preuss doit être rejeté, mais que ce genre peut être accepté au sens de Saccardo ; on peut discuter si les espèces à conidies toujours sub-hyalines sont des Acrothecium au sens de Preuss. Mason range le champignon étudié par lui dans le genre Acrothecium ( $A$. lunatum Wakk.) à cause du petit diamètre de la première moitié des conidies ; cependant il est possible que $A$. lunatum soit un Acrotheciella Koorders (conidies courbes avec cellules de différents diamètres). Mason remarque en outre que $A$. multisporum Preuss a été à tort rejeté du genre Acrothecium et placé par Saccardo dans le genre Acrotheca.

L'espèce type du genre serait naturellement l'Acrothecium multisporum Preuss, qui selon Mason et contrairement à l'opinion de Saccardo est un vrai Acrothecium et non un Acrotheca.

Nous acceptons les conclusions de Mason et nous admettons provisoirement le genre Acrothecium Preuss emend. Sacc. p. p. nec Corda (sous-genre). Ce genre renferme une vingtaine d'espèces, toutes saprophytes, vivant presque toutes sur végétaux morts. Saccardo a divisé le genre Acrothecium en deux sous-genres ; Eu-Acrothecium Sacc. avec conidies fuligineuses, et Acrothecula Sacc. avec conidies sub-hyalines. Notre espèce occupe une position intermédiaire entre ces deux sous-genres, dont elle constitue le trait d'union, puisque les conidies, hyalines dans la jeunesse, sont généralement en partie obscures et presque toujours foncées dans la cellule centrale. Nous croyons que ce caractère, dont nous n'avons rencontré aucun exemple dans les autres espèces d'Acrothecium, est suffisant pour distinguer notre champignon.

Notons en passant que la coloration des cellules médianes des conidies est presque aussi sombre que dans d'autres Dématiées ; par exemple, dans le Brachysporium coryneoideum (De Notaris) Saccardo, un ancien Helminthosporium avec conidies ovoïdes ou piriformes; ce genre diffère seulement d'Acrotheca par ses conidies solitaires et non agrégées. Cette particularité est facile à reproduire dans notre souche et on peut accentuer la production des conidies solitaires en cultivant le champignon dans des milieux 
pauvres ou peu favorables (particulièrement acides). On peut arriver ainsi à la production de formes presque monoconidiennes. Dans ce cas, notre champignon se rapproche du genre Brachysporium Sacc. et, en fait, quelques espèces, comme le B. coryneoideum (De Notaris) Saccardo ressemblent beaucoup à notre souche, pour la forme et la coloration des conidies, comme nous l'avons déjà noté.

Le champignon étudié présente encore beaucoup d'affinités avec les Spondylocladium Mart., genre très voisin des Acrothecium, mais dont les conidies sont disposées sur plusieurs plans (conidies pleurogènes) ; nous avons déjà indiqué que notre espèce a des conidies acro-pleurogènes, quoique plutôt disposées en capitules terminaux ; cependant elles sont nettement pleurogènes dans les cultures riches. Notre espèce diffère par les conidiophores des Napicladium Thuemen, quoique les différences ne soient pas toujours aussi marquées qu'en théorie.

Comme dans le genre Acrothecium il y a des espèces avec conidies sub-hyalines, faut-il accepter la fusion des genres Cacumisporium (conidies obscures) et Acrothecium? Mason en doute quoique sans se prononcer en définitive ; nous-même doutons qu'un genre avec conidies constamment sub-hyalines puisse être confondu avec un genre à conidies toujours foncées; et cela en dehors d'autres différences secondaires, comme la question de la non-validité du nom générique Acrothecium. Cependant, suivant l'exemple de Mason, en attendant que l'on puisse réviser assez complètement les espèces de ces genres, nous conservons le genre Acrothecium au sens de Saccardo. Quoique n'ayant pu effectuer la comparaison des espèces d'Acrothecium jusqu'ici connues avec notre souche sinon par les diagnoses, nous croyons pouvoir la distinguer par sa position intermédiaire entre les deux sous-genres : Eu-Acrothecium et Acrothecula (quoique nous pensions que ce cas n'est pas isolé, étant donné que presque toutes les autres espèces n'ont pas été complètement étudiées), par la coloration centrale plus foncée des conidies et surtout par son habitat très particulier (1).

(1) Nous croyons que si vraiment on peut fusionner les deux genres Acrothecium et Cacumisporium ce sera le nom générique Cacumisporium qui devra être substitué à Acrothecium; dans le cas contraire, on pourra accepter Acrothecium Preuss emend. Saccardo p. p. conf. Mason nec Corda avec comme espèce type A. multisporum Preuss ou mieux encore abandonuer définitivement le nom générique Acrothecium, deux fois incorrectement employé par les deux premiers auteurs.

Pour plus de clarté, nous pouvons ainsi résumer les caractéristiques des genres jusqu'ici nommés, plus ou moins voisins d'Acrothecium :

I. Gonidies continues................... Acrotheca Fuck.

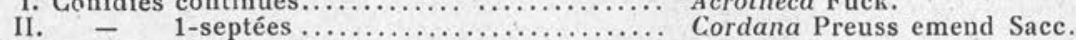

III. - 2-pluri-septées.

A. Conidies verticillées

1. Conidies acrogènes. 
Voici la diagnose de la nouvelle espèce que nous établissons ad interim :

Acrothecium nigrum Cif., n. sp. ad interim(1).

(In agaro, Sabouraud dicto, culto). Colonia lanosa, grisea usque nigra, densa; agaro nigro-pigmentato; mycelio repente vel sub-repente, fusco crassoque vel hyalino aut laete colorato, tenue, chlamydosporis intercalaribus, monilioideis, plus minus longe catenulatis efformante ; conidiophoris simplicibus vel parce ramosis, erectis vel suberectis, sub-hyalinis aut fuscidulis, apice læte coloratis, breviter denticulatis, capitulo nigro, acrogeno vel acro-pleurogeno conidiorum ornatis; conidiis rarius singulis, sæpe 2-20 capitulatis, continuis vel 1-3 septatis, consuete 3-septatis, piriformibus, ellipticis, ovoideis curvulis vel irregularibus, initio subclavulatis, continuis, subhyalinis, dein septatis, fuscis, loculis interioribus fuligineis usque nigris, extimis minoribus, fuscis usque subhyalinis, basi acutato-truncata, $16-30 \times 6-11 \mu$, consuete $22-26 \times 8-9 \mu$, promedio $24 \times 8 \mu$.

Habitat : In epidermide humana, a morbo «pinta 》 dicto infecta. Iguala (Estado de Guerrero), Mexico, leg. et comm. I. Ochoterena. A cœeteribus Acrothecium, præcipue habitu, conidiis hyalinis dein fuscis, loculisque intermediaribus conidiorum nigris, terminalibus dilute fuscis, differt. Characteribus Montoyellæ nigræe Cast. subaffinis.

Nous n'avons jamais vu d'autre forme que la forme conidienne. On ne connaît de forme ascosporée que pour une espèce, l'Acrothecium anixiae v. Hoehnel, qui a décrit l'état métagénétique parfait, quoique sans confirmation expérimentale (Anixia bresadolae v. Hœhnel, Périsporiacées Perisporiées Hyalosporées) (2).

Etiologie et pathogénicité. - Nous ne savons rien sur ces points. Le champignon était associé à une espêce levuriforme 'qui sera bientôt publiée.

a. Conidies sub-hyalines...

b. Conidies sombres.

2. Conidies acro-pleurogènes.

a. Conidies courbes, avec la cellule médiane

b. Conidies droites, avec les cellules à peu près égales..............................

B. Conidies non verticillées

1. Conidiophores courts et mous ............ Napicladium Thuem.

2. Conidiophores longs et assez rigides........ Brachysporium Sacc.

(1) Nous nommons cette espèce nigrum car ce nom n'est pas représenté dans les Acrothecium que nous connaissons. Dans le cas où Montoyella nigra Cast. serait le même champignon, le passage du nom spécifique sera plus facile, et il n'y aurait pas l'inconvénient d'un synonyme spécifique en plus.

(2) LINDAU considère cette espèce comme la forme conidienne de l'Anixia myriasca von Hoehnel; c'est une simple erreur de transcription, car von Hoenel déclare explicitement le contraire. D'autres espèces ont été trouvées associées avec des Sphériacées, par exemple l'Acrothecium simplex B. et Br., l'A. atrum Preuss, etc. 


\section{Affinités de ce champignon avec les autres parasites du (( pinto ).} - Si nous nous adressons à la systématique des espèces trouvées dans le " pinto " noir (et aussi dans le " pinto " en général) nous rencontrons plus d'incertitudes encore. Castellani (in Castellani et Chalmers) a établi un genre nouveau, Montoyella, avec deux espèces $M$. nigra Cast. et $M$. bodini Cast. dont la première seule existe dans le " pinto noir. Castellani et Chalmers indiquent que ce genre est semblable aux Microsporum et Trichophyton et produit des conidies et des fuseaux. Dans le même traité, les deux auteurs placent le genre Montoyella parmi les Trichophyton avec filaments mycéliens et spores dans les lésions, donnant en culture des hyphes conidifères avec spores latérales seulement. Et ensuite : "Genre provisoire. Deux types d'hyphes mycéliennes ; quelques-unes minces, ramífiées, septées; d'autres plus épaisses avec beaucoup de chlamydospores intercalaires. Des filaments épais partent des hyphes délicates avec de grandes conidies piriformes ou globulaires. 》Et après, à propos de Montoyella nigra : "Colonies noires sur gélose maltosée; noires aussi sur gélose glycérinée. \D'après Brumpt nous savons qu'il y aurait une troisième espèce de Montoyella, la $M$. bodini Urueta dans le caraté rouge : que le caraté noir est peut-être la « tinea nigra 》 due au Cladosporium mansoni, et surtout que "l'absence de recherches mycologiques ne permet pas encore d'identifier la «pinta » ou «mal del pinto » du Mexique » (1).

N'ayant pu lire les travaux originaux de Castellani sur le genre Montoyella, ni les autres travaux mycologiques sur les espèces du même genre, nous avouons qu'il est très difficile d'indiquer les affinités qu'il y a entre $M$. nigra et notre Acrothecium. Les points d'identité sont :

1. Culture très noire sur maltose (et aussi sur d'autres milieux).

2. Production d'un pigment noir partiellement diffusible dans la gélose.

3. Deux types de mycélium dans les cultures, l'un mince et clair, l'autre épais et foncé.

4. Production abondante de chlamydospores dans les cultures.

5. Conidiophores avec conidies terminales.

6. Forme en poire des conidies.

Mais comme il n'y a pas d'indications sur le cloisonnement des conidies et sur leur façon de s'agréger, le doute subsiste. La différence fondamentale est la présence des fuseaux et l'assimilation

(1) Selon Castellani et Chalmers c'était aussi l'opinion de Montoya que le « caraté colombien est différent du « pinto » mexicain. 
de ce champignon aux Trichophyton. On pourrait comparer les conidies septées aux fuseaux, mais la signification en est très différente. Par conséquent, quoique émettant l'hypothèse que Montoyella nigra est peut-être la même chose que notre Acrothecium, nous sommes dans l'obligation de penser qu'il s'agit d'une espèce nouvelle.

Observation sur le pigment de l'Acrothecium nigrum. - L'étude du pigment formé par ce champignon eût été d'un grand intérêt ; mais comme elle sort de notre champ d'étude, elle a été à peine ébauchée (1). Nous avons préparé de la gélose pepto-glycosée selon la formule usuelle, ayant la précaution de décolorer le bouillon avec du charbon animal. La gélose était d'une couleur paille très claire ; desséchée, elle ne colorait pas l'eau, même après une longue ébullition. La gélose fut distribuée dans trois grandès boîtes de Petri, de façon à former la plus mince couche possible, et ensemencée avec le champignon.

Celui-ci ne couvrit pas complètement la surface libre, car l'évaporation desséchait rapidement la gélose. Les colonies furent séchées complètement dans l'étuve à paraffine, jusqu'au détachement de la pellicule de gélose. Sur ce complexe (gélose et champignon) nous avons effectué les essais ci-dessous.

Dans l'eau distillée on voit diffuser lentement un pigment noir, avec une nuance presque pourpre, mais très pâle. Le pigment se dissout plus rapidement à l'ébullition, et la solution est un peu plus foncée. L'extraction n'est que partielle, car, même après une ébulJition de plusieurs heures, la gélose est toujours presque aussi noire qu'au commencement ; probablement, la portion soluble dans l'eau est très limitée. La solution aqueuse, d'abord noire avec une nuance pourpre (rappelant la couleur très diluée des noirs « chauds »), vire après quelques heures au noir jaunâtre; la couleur est ŝnalement très semblable à celle de la solution alcaline. L'eau acidulée avec les acides acétique ou sulfurique ne dissout pas la couleur en quantité appréciable. Par contre l'eau alcalinisée avec de la soude $(10 \mathrm{p} .100)$ dissout très rapidement le pigment même à froid, mais seulement en petites proportions. L'eau salée dissout un peu le pigment.

Les essais spectroscopiques ont été effectués soit avec la solution aqueuse, soit avec la solution alcaline, directement ou après dialyse pour séparer la gélose dissoute dans l'eau. I'examen spectroscopique (au microspectroscope) de la solution aqueuse brute

(1) Nous avons le plaisir de remercier le Dr E. Balzarotti, chimiste de la Station, qui a collaboré activement à l'exécution de ces essais 
nous a révélé une absorption très faible sur presque tout le spectre, sauf dans l'orange et le rouge, mais sensible dans le violet, et presque complète dans l'extrême violet; la portion d'onde plus large du violet est absorbée, et l'absorption est toujours plus faible en passant du violet au rouge.

La solution alcaline (dans la soude) diluée détermine une absorption presque égale dans le violet, moindre dans le bleu et faible dans le vert; rien dans les autres fractions du spectre. Cette solution se décolore petit à petit, lorsqu'elle est exposée à la lumière vive du jour.

Nous n'avons pas effectué l'étude du pigment noir dans d'autres solvants.

\section{RÉSUMÉ}

Ce travail est consacré à l'étude morphologique et biologique d'un champignon isolé au Mexique dans un cas de «pinta negra 》 et rapporté au genre Acrothecium Preuss, emend. Sacc. nec Corda. Il est possible que ce champignon soit voisin de Montoyella nigra Cast., ou même identique à cette espèce. Mais les documents dont on a disposé n'ont pas permis une comparaison complète. Il est donc décrit au moins provisoirement comme espèce nouvelle, sous le nom d'Acrothecium nigrum, n. sp. ad interim.

\section{BibLiographie}

Castellani (A.) et Chalmers (J.). - Manual of tropical medecine, 3e édit., 1919, p. 1023.

Conda (A.-C.-J.). - Icones fungorum, II, p. 10, pl. IX, fig. 49, 1838.

Von Höhnel (F.). - Fragmente zur Mykologie. I Mitth., $\mathrm{N}^{\circ} 2$. Sitzungsb, d. m. mathem. naturw. Kl., CXI, 1912.

Lindau (G.). - in Rabenhorst's Kryptogamen Flora. 2e édition, 1910, IX, p. 143-149,.

Mason (E.-W.). - Annotated account of fungi received at the Imperial Bureau of Mycology, List II, Fasc. 1, pag. 1-43, 1928.

Preuss (F.). - Hoyersw. No 36, in Linnaea, XXIV, 1851, pag. 111 ; in Sturm's, Deutsch. I., 3, VI, Heft 30, tab. 43, 1851.

Saccardo (P.-A.). - Michelia, 11, pag. 29, 1882.

Saccardo (P.-A.). - Sylloge fungorum omnium hucusque cognitorum, etc., IV, 1886.

Station Nationale Agronomique et Collège d'Agriculture de Moca (République Dominicaine). 